\title{
Norois
}

Environnement, aménagement, société

\section{Les tensions fondatrices du développement local autour du restaurant Bras à Laguiole : " centralité ", qualité et créativité}

The founding tensions of local development around Bras restaurant in Laguiole:

"centrality", quality and creativity

\section{Olivier Etcheverria}

\section{OpenEdition \\ Journals}

\section{Édition électronique}

URL : https://journals.openedition.org/norois/3595

DOI : $10.4000 /$ norois.3595

ISSN : $1760-8546$

\section{Éditeur}

Presses universitaires de Rennes

\section{Édition imprimée}

Date de publication : 30 juin 2011

Pagination : $57-71$

ISBN : 978-2-7535-1479-9

ISSN : 0029-182X

\section{Référence électronique}

Olivier Etcheverria, «Les tensions fondatrices du développement local autour du restaurant Bras à Laguiole : "centralité », qualité et créativité », Norois [En ligne], 219 | 2011, mis en ligne le 30 septembre 2013, consulté le 14 janvier 2022. URL : http://journals.openedition.org/norois/3595; DOI : https://doi.org/10.4000/norois.3595

Ce document a été généré automatiquement le 14 janvier 2022.

(c) Tous droits réservés 


\title{
Les tensions fondatrices $\mathrm{du}$
} développement local autour du restaurant Bras à Laguiole : " centralité », qualité et créativité

\author{
The founding tensions of local development around Bras restaurant in Laguiole: \\ "centrality", quality and creativity
}

\section{Olivier Etcheverria}

1 La singulière trajectoire du plateau de l'Aubrac montre le cas d'un espace rural, hâtivement qualifié de "marge » (Collectif, 1970), qui présente un développement socio-économique récent à contresens des évolutions prévues pour les zones de faible densité. En effet, il se caractérise par sa résistance à la désertification, par son usage social original et dynamique du territoire et par sa vitalité nonobstant son isolement géographique.

2 L'un des puissants ressorts de cette vitalité est, semble-t-il, le (grand) restaurant ${ }^{1}$ par l'ouverture vers l'extérieur qu'il assure et le rayonnement international qu'il permet. En se positionnant comme un acteur "central», Michel Bras a progressivement construit une relation durable entre le local et le global, essentielle à la qualification d'atouts et à la valorisation d'attraits (en particulier touristiques). Ce cas tendrait à montrer que ce qui est déterminant n'est pas le caractère plus ou moins « marginal » de l'espace géographique d'appartenance mais l'existence d'un acteur "central», charismatique, à dimension internationale, constructeur à la fois d'une image (hospitalière et attrayante) et d'un discours (sur les valeurs de la qualité et de la créativité) et porteur d'un projet de territoire capable d'impulser une dynamique localisée et de mettre en œuvre les conditions nécessaire au développement local: le cuisinier. La « centralité » de Michel Bras est également lisible dans la capacité qu'il a montrée à se positionner judicieusement dans le système local d'acteurs et à articuler sa stratégie individuelle aux dynamiques socioéconomiques à l'œuvre. 
Cette réflexion sur le " pouvoir développant » du restaurant en milieu rural s'inscrit dans le cadre élargi du devenir des espaces ruraux. L'hypothèse développée ici n'a pas fait l'objet de travaux de recherches, ni de controverses scientifiques. Il s'agit donc de démontrer, à partir d'un exemple supposé révélateur et significatif, celui du restaurant Bras à Laguiole, sur le versant aveyronnais du plateau de l'Aubrac, triplement étoilé au guide Michelin, la capacité d'un restaurant à constituer l'un des leviers déterminants du développement local en espace rural.

4 La démarche méthodologique directe et participante choisie a consisté, d'une part à rencontrer Michel Bras une première fois en octobre 1999, année de l'obtention de la troisième étoile au guide Michelin, puis une dizaine de fois ensuite, et à découvrir (et redécouvrir) sa cuisine. Parallèlement, une trentaine d'entretiens a été réalisée auprès des habitants, des producteurs et des acteurs du tourisme afin de cerner leurs rapports réels et idéels au restaurant et leurs articulations au lieu. Ces enquêtes de terrain visaient à appréhender les retombées spatiales, socio-économiques et culturelles d'un restaurant sur le développement local en espace rural.

\section{Michel Bras en Aubrac}

\section{Un long et viscéral ancrage au plateau}

5 Michel Bras est un cuisinier viscéralement attaché à «son » plateau, profondément animé par l'amour du lieu et de ceux qui l'habitent. Natif d'un petit village aveyronnais, il grandit auprès d'un père forgeron et d'une mère cuisinière. Le cuisinier va ainsi acquérir progressivement et durablement, de l'intérieur, une connaissance fine et précise du lieu et du groupe social qui le met en valeur.

6 Après un apprentissage de la cuisine auprès de sa mère, Michel Bras reprend en 1978 à son compte l'exploitation du petit restaurant familial Lou Mazuc², blotti dans le bourg de Laguiole. Fort de ce long ancrage au lieu, il va construire culinairement son «alliance du Bon \& du Beau » : «Apprivoiser l'Aubrac a été un long cheminement. De ce lieu habité par le silence, saturé de lumière, nourri de ses saisons immuables, renouvelées inlassablement, j'ai acquis cette relation de chaque instant à l'émerveillement; sons, couleurs, sent-bon, tout est Beau \& Bon » (Bras dans Fumey et Etcheverria, 2004). Le cuisinier tient le «Beau » de son père dont il héritera la rigueur et qui le conduira à voyager jusqu'au Japon où il sera à l'initiative d'une collection de couteaux «haute couture ». Il tient le «Bon" de sa mère qui lui inculqua les bases techniques culinaires et avec qui il voyage dans le patrimoine culinaire du lieu. Cette esthétisation du goût est autorisée par la compréhension de sa relation au temps et à l'espace: "Alors, alors seulement, grisé, sous le charme, le Beau \& le Bon me reviennent à la mémoire. L'esprit et le corps régénérés, je redeviens pleinement sensible. J'ai le profond sentiment que le Plateau m'a offert l'aptitude à m'ouvrir au Monde. Je suis ensorcelé par l'Aubrac ${ }^{3}$.»

7 En quelques années, le rayonnement du restaurant dépasse les limites du plateau pour se diffuser à l'échelle nationale puis internationale, en direction de l'extrême orient notamment. Dès le milieu des années 1980, le restaurant est distingué et récompensé par le guide Gault \& Millau qui lui attribue la note de 15/20. Le succès se confirmant, à l'étroit, Michel Bras décide, en 1992, de s'installer à six kilomètres, à l'écart du bourg. Il choisit alors d'implanter son nouvel hôtel-restaurant au sommet du Puech de Suquet, 
isolé et battu par l'écir ${ }^{4}$. Selon son discours, cette stratégie spatiale originale constitue un choix esthétique et philosophique de vie. C'est l'expression identitaire du droit à vivre et cuisiner là. « Nous avons le goût de l'Aubrac. Nous sommes nés sur son plateau, nous y avons passé notre enfance, heureuse, et nous y travaillons. Nous y trouvons notre raison de vivre et notre inspiration. L'Aubrac, c'est notre creuset : c'est sur ce lieu que convergent pour nous toutes les forces de la création; là que s'est aiguisé notre regard, là que s'est affûtée notre oreille, que se sont forgés nos sens de langue et de nez, là que nos mains ont appris à toucher. Comme les cascades, les lacs, les hêtres, les troupeaux même, nous sommes nés de l'Aubrac. Nous en avons la texture, la carrure, le bouquet... et la chanson ${ }^{5}$.»

\section{Le nouveau restaurant comme signal architectural}

8 Le nouveau restaurant, adossé à un hôtel de 12 chambres permettant d'héberger une clientèle touristique nationale et internationale, va constituer un réel signal architectural sur le plateau. Sa conception qui est confiée au designer Eric Raffy repose sur la recherche d'une osmose entre les paysages mamelonnés du plateau et une architecture de métal, de granit et de verre qui épouse les formes du Puech du Suquet (photo 1). L'hôtel-restaurant est ainsi composé de 3 bâtiments longilignes, qui constituent des métaphores des burons, orientés perpendiculairement à la draille d'accès, ancienne voie de transhumance et chemin de l'église (fig. 1). Grâce aux grandes baies vitrées, véritables vitrines des pâtures où paissent les vaches de la race Aubrac, le restaurant est une fine membrane qui abrite mais aussi dévoile les paysages dans un rapport dialectique intérieur/extérieur.

9 Certes, le parti de l'intégration paysagère est affirmé et affiché : «Le corps principal restaurant, accueil et salon - est une forme basaltique longiligne qui jaillit de la terre, biaise et massive, buron retrouvé. Elle est le toit, elle flotte sur un soubassement de verre, qui la rend aérienne, contrastant avec son origine souterraine, amenant une conscience de jaillissement. La salle de restaurant s'ouvre sur l'extérieur, permettant à la lumière de se glisser. La partie salon en son extrémité aérienne est totalement vitrée, irréelle. Les chambres sont basées sur le même principe, pour ne constituer qu'une architecture. La plupart sont en rez-de-nature. Un décaissé du terrain préserve l'intimité de chaque chambre, et donne à chacun des occupants un Aubrac qui semble être pour eux seuls. La chambre, la salle d'eau sont ouvertes sur l'extérieur par de larges baies. Elles sont aménagées pour une communication parfaite avec les odeurs, les fleurs, les gazouillis... Ces chambres tantôt voisinent avec le ciel, tantôt s'impriment du jardin, des espaces de l'Aubrac. L'évasion est garantie. De ce lieu que nous avons voulu magique, l'Aubrac se met en scène par des points de vue, des cadrages pour le plaisir de tous nos sens. Un Aubrac qui nous rassure sur les véritables valeurs du Bon \& du Beau ${ }^{6} . »$ Cependant, posé au sommet du Puech du Suquet, en position dominante, l'hôtel-restaurant constitue un signal, visible et repérable de loin, à plusieurs dizaines de kilomètres à la ronde. Ainsi, si le plateau de l'Aubrac constitue une «marge » en termes d'organisation et de dynamique des espaces géographiques, Michel Bras devient un acteur « central » de celle-ci. 
Photo 1 : L'hôtel-restaurant Bras : un signal architectural (cl. O. Etcheverria)/The hotel-restaurant Bras : an architecture signal

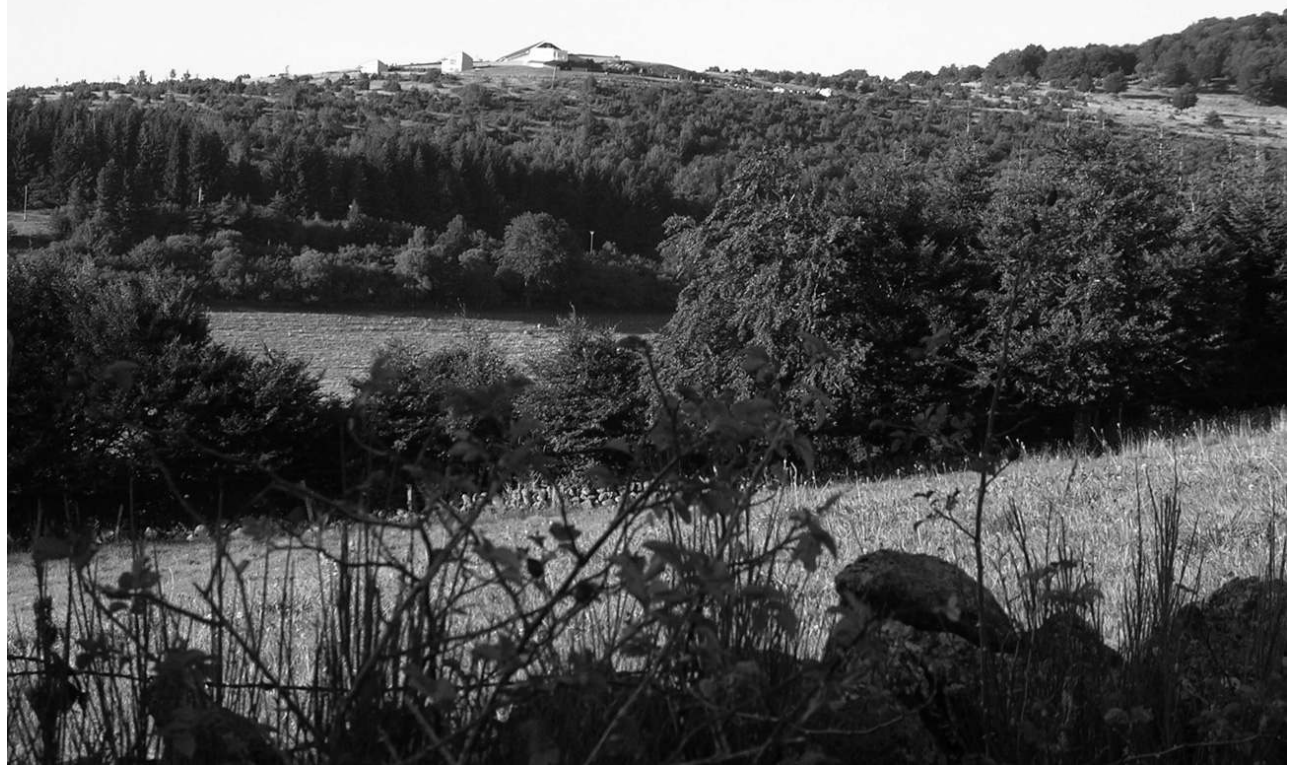

Figure 1 : Trois métaphores des burons organisés perpendiculairement à la draille qui est symboliquement orientée vers le clocher de l'église de Laguiole (source M. Bras)./Three metaphors of " burons" (sheds where shepherd make cheeses in Aubrac plateau) organized at right angles to the "draille" (mountain pasture path) which is symbolically oriented to the church tower of Laguiole

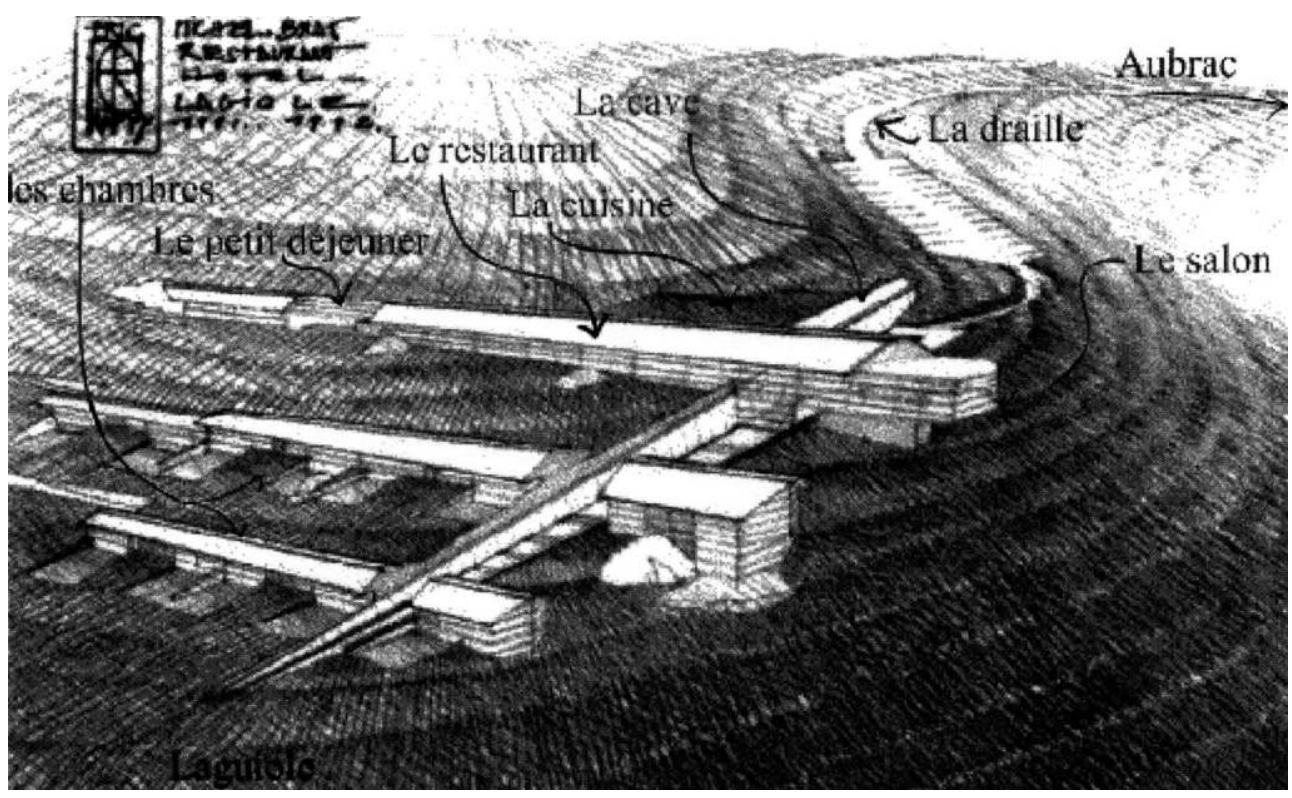

\section{Un cuisinier « central »}

10 Plus que le lieu qu'il cherche, par le truchement de l'assiette, à valoriser et à afficher, c'est la personne même de Michel Bras qui est progressivement devenue «centrale ». 
D'une part, il se positionne au centre d'un ensemble de forces centrifuges et centripètes constituées de flux de personnes, de marchandises, d'idées, de capitaux, d'initiatives et de savoir-faire. D'autre part, à travers la dimension touristique de son établissement et l'échelle nationale-internationale de ses filières d'approvisionnement (au moins pour quelques produits très spécifiques), il a construit un lien durable entre le local et le global. Enfin, grâce à la diffusion d'une image hospitalière et attrayante et d'un discours fondé sur les valeurs de la qualité et de la créativité, il a initié une ouverture vers l'extérieur et encouragé un rayonnement à l'échelle internationale (notamment par ses activités de conseiller culinaire dans un restaurant d'hôtel à Toya, au Japon). Il est en effet à l'origine de formes de mobilités touristiques, volontaires, choisies et motivées. Durant les sept mois d'ouverture de l'hôtel-restaurant, de début avril à fin octobre, le cuisinier sert ainsi environ 45000 couverts.

11 Michel Bras est également un cuisinier « central » par les caractéristiques de sa cuisine à la fois fortement ancrée et nourrie d'influences extérieures, auxquelles il s'abreuve durant ses voyages professionnels et ses séjours touristiques internationaux. Il s'agit d'une cuisine ancrée car elle ne peut être pleinement dégustée et appréciée qu'in situ (Etcheverria et Bras, 2004). C'est au contact et en contemplation devant sa terre natale que Michel Bras trouve son inspiration: "C'est en écoutant le réveil de l'Aubrac au printemps que j'ai imaginé mon gargouillou de jeunes légumes? ${ }^{7}$. Et en arpentant les montagnes, je suis tombé nez à tige avec une reine-des-prés. Son parfum de miel et d'amande m'a enivré. » Ainsi, la composition des assiettes repose sur une ressemblance visuelle plateau/plat: "Mes assiettes racontent une histoire, retracent une démarche intuitive. Je veux qu'elles témoignent des paysages, des roches, des ciels et de la lumière qui font ma vie.» Cette ressemblance plateau/plat est imposée et découle d'une connaissance kinesthésique et idéologique du lieu (photos 2 et 3). Concomitamment, il s'agit d'une cuisine qui est capable de digérer de nouvelles saveurs découvertes à l'occasion de nombreux séjours en Orient (Inde) et en Extrême-Orient (Japon) notamment. Selon le cuisinier, c'est l'ubiquité de l'Aubrac qui autorise cette "digestibilité »: «J'ai le profond sentiment que [ce] plateau m'a offert l'aptitude de m'ouvrir au monde. Je sais aujourd'hui que l'Aubrac donne accès à l'universel. Partout, il y a ce petit coin, cette parcelle, ce muret de pierre sèche qui me transporte dans un voyage de l'imaginaire " (Bras, 1999). Le plat intitulé Mémoire de voyage en terre "épicée ", le foie gras de canard au naturel, ponctué de pomme-poivre, de carotte-curry, de betteraveframboise en témoigne.

12 La centralité de Michel Bras réactive la question d'une part de son positionnement relatif dans le système local d'acteurs et d'autre part de l'articulation de sa stratégie individuelle avec les dynamiques socioéconomiques à l'œuvre montrées par L. Laurens (1999) et J. Bessière (2001). 
Photo 2 : Une portion du plateau de l'Aubrac à la fin de l'été (cliché de l'auteur pris le 10 septembre 2009 dans les environs proches de Laguiole)/A part of Aubrac plateau at the end of summer (picture taken by the author on 10 September 2009 in the immediate vicinity of Laguiole)

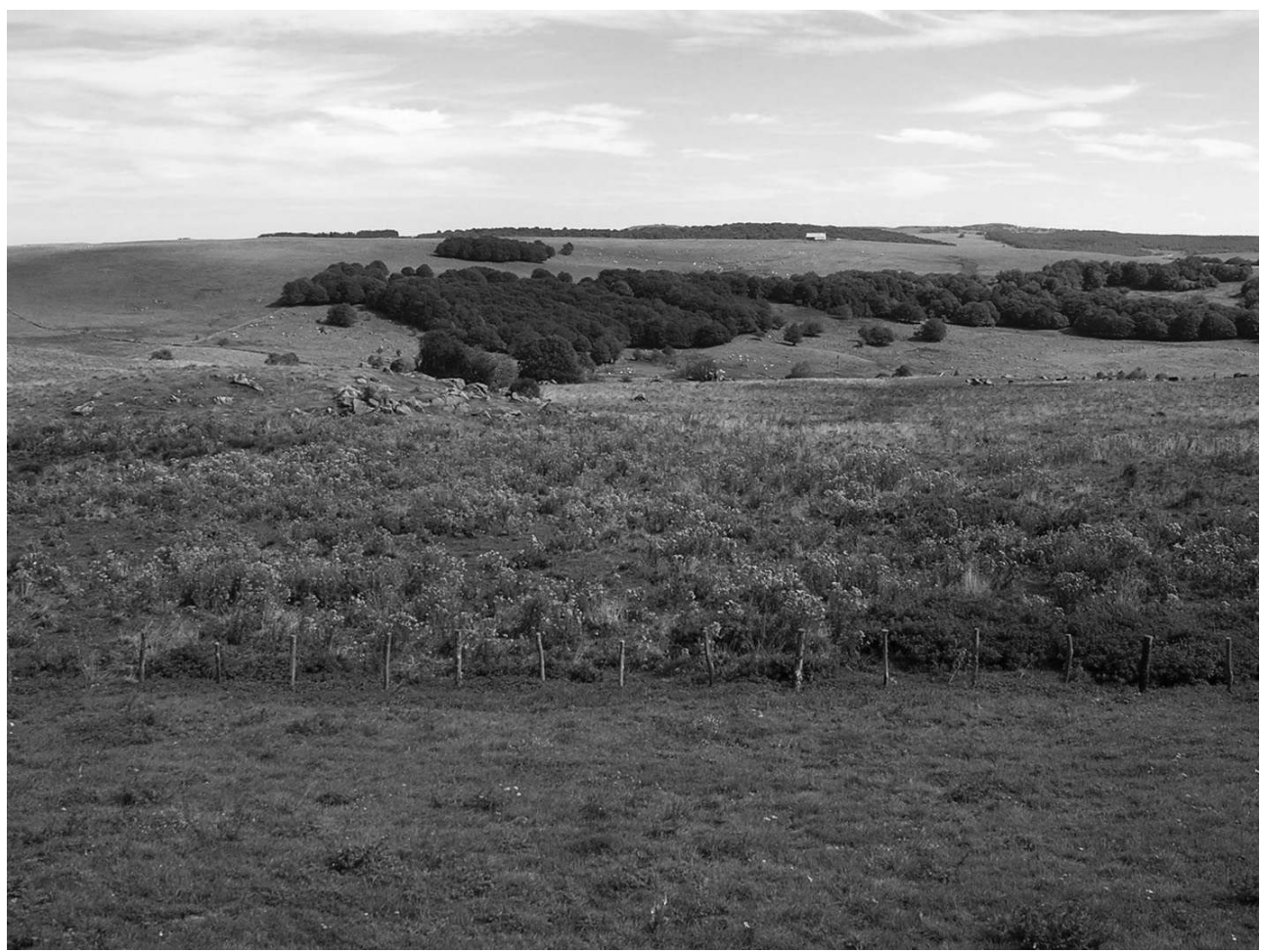

13 Photo 3: Un plat de fin d'été: De Méditerranée, le maquereau juste cuit, un assaisonnement à la feuille de roquette et à la fleur de sureau; ficoïde, chicorée \& pourpier vert (cliché de l'auteur pris le 10 septembre 2009 au dîner)/An end-summer dish: From Mediterranean, just done mackerel, a seasoning with rocket leaves \& elderflower; crystalline ice plant, curly endive \& green purslane (picture taken by the author on 10 September 2009 at dinner) 


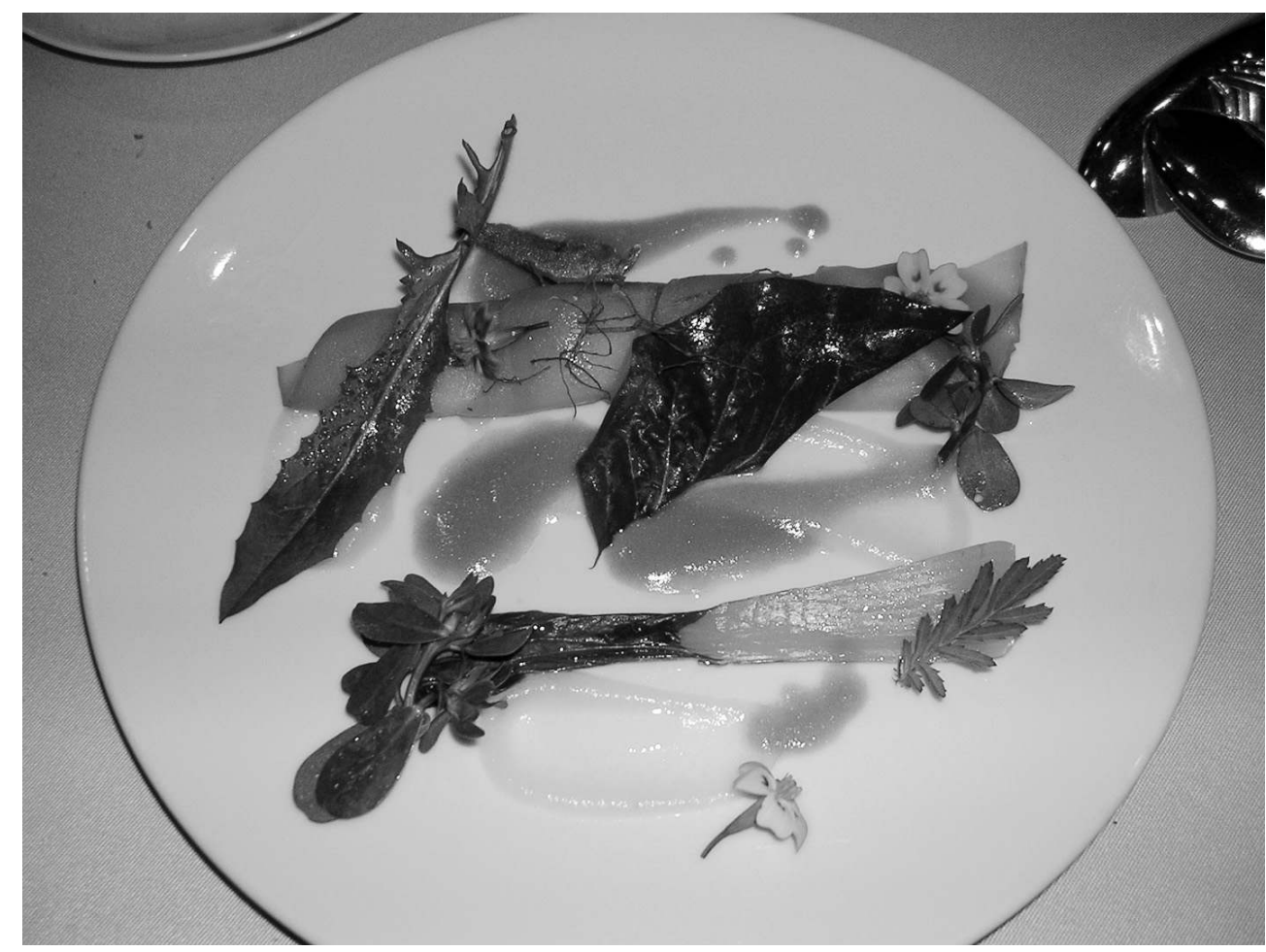

\section{De la pratique individuelle d'un cuisinier dans la dynamique de mise en place des conditions nécessaires au développement local : images et discours à l'œuvre}

Par la construction progressive et la diffusion à une échelle internationale d'une image hospitalière et attrayante et d'un discours fondé sur la qualité et la créativité, la compréhension du passage de l'initiative individuelle de Michel Bras à sa contribution à la dynamique de mise en place des conditions nécessaires au développement local est lisible à deux niveaux. D'une part, le changement radical des qualités du lieu qui va influencer les pratiques et les représentations sociales des habitants permanents mais aussi, et surtout, temporaires (les touristes). D'autre part, l'encouragement, l'affichage et la visibilité extérieure donnés à la transformation qualitative et créative des pratiques des autres acteurs socio-économiques locaux.

\section{Changer radicalement les qualités du lieu}

Grâce à l'ouverture de son nouvel établissement, accueillant, confortable, largement ouvert vers l'extérieur mais aussi "protecteur", Michel Bras va, par mimétisme, rendre le plateau, à l'image du restaurant, hospitalier et attractif. L'Aubrac fut longtemps un espace très inhospitalier. Il s'agit en effet d'un plateau d'altitude (qui constitue la plus méridionale des zones volcaniques du Massif Central) et de faible densité de population aux conditions de vie particulièrement rudes et difficiles. "Dans l'ensemble de l'Aubrac, les hivers sont longs et rigoureux. Les premières chutes de neige peuvent survenir à la fin d'octobre, les dernières à la fin de mai. Le vent accumule parfois le tapis floconneux en congères épaisses. La "tourmente", tempête de neige 
portée par les souffles venus du nord-ouest qui aveugle et désoriente, a fait périr jadis plus d'un voyageur égaré malgré les hautes balises de granit bordant les chemins (Barbey, 1991).» Il s'agissait alors d'un espace répulsif qui constitua une terre d'émigration. En effet, dès le XvIII ${ }^{e}$ siècle, l'Aubrac connait une hémorragie humaine qui conduit les natifs vers l'Espagne, le sud de la France et, surtout, Paris où ils deviennent bougnats et limonadiers dans des cafés-charbons. Progressivement, le vieillissement démographique et l'archaïsme agricole (production fromagère décrépie, race bovine quasiment disparue et déclin des estives) expliquent l'effondrement socio-économique du territoire dès les années 1950. Une enquête du CNRS ${ }^{9}$ menée entre 1960 et 1964 établissait un cruel diagnostic de crise et prédisait la condamnation du pays et la disparition des activités « traditionnelles ».

Le cuisinier va alors participer de manière originale à la dynamisation et à l'attractivité touristique du plateau en activant-réactivant le souvenir de l'hospitalité nourricière légendaire des moines de la Dômerie d'Aubrac qui accueillent, depuis le Moyen Âge, des pèlerins en route vers Saint Jacques-de-Compostelle. Ces moines ont développé l'habitude de signifier l'hospitalité en proposant de partager l'aligot: préparation à base de pommes de terre écrasées et de crème fraîche enrichie de tomme fraîche de Laguiole $^{10}$. L'appellation trouve son origine dans le mot latin aliquid qui signifie "quelque chose à manger ${ }^{11}$ ». Dans son Dictionnaire, H. Affre (1903) revient sur l'étymologie du mot : "Ce mot français d'origine moderne a la même signification que les mots patois oligot, oligouot qui désignent un mets particulier aux montagnes de Laguiole. Les buronniers ou cantalès excellent dans sa préparation. C'était le plat obligé de tout festin de noces et, dans ce cas, particularité curieuse à noter, la préparation n'était pas le fait de la maîtresse de maison ou de sa servante, mais de deux convives du sexe fort qui, experts en la matière, quittaient la table au moment voulu et passaient à la cuisine pour prendre en main, avec tous les soins désirés, la confection de cette partie de menu. La recette de ce mets, qu'on chercherait vainement dans « le parfait cuisinier » est des plus simples. Dans une poêle à frire bien lavée et bien frottée, on met une quantité convenable de beurre frais et lorsque, sur un feu clair et régulièrement entretenu, le beurre est fondu, on prend des pommes de terre préalablement cuites à l'eau qu'on dépose dans le beurre et qu'on écrase jusqu'à réduction. Cela fait, on prend, en quantité égale aux pommes de terre, de la tomme ou fromage encore imparfait qu'on divise en tranches aussi minces que possible et qu'on met à son tour dans la poêle. Le tout est ensuite salé convenablement, assaisonné d'ail et de persil hachés, et remué sans cesse et dans tout les sens jusqu'à ce que le mélange soit complet. Pour faciliter cette dernière opération, on ajoute un peu d'eau chaude si c'est nécessaire. On reconnaît que la cuisson est suffisante et l'aligot prêt à être servi parce que la matière est devenue filante comme la fourme ou le gruyère dans une soupe bien mitonnée. Les anciens, qui ne connurent pas le pomme de terre, faisaient usage de tranches minces de pain blanc, appelées en patois pa de micho. " L'aligot demeure un plat fade comme en témoigne G. Subervie (1953) : "L'aligot est un mets qui ne laisse personne indifférent : ou on l'aime et alors on ne tarit pas d'éloges sur son goût, sa délicatesse, son onctueuse tenue; ou on ne l'aime pas et l'on s'indigne de penser qu'on puisse accepter comme un régal cet insipide plat de colle à pâte, sans saveur, sans odeur et sans grâce. » Mais, il est roboratif. Michel Bras perpétue cette habitude hospitalière en proposant encore aujourd'hui l'aligot à sa carte sous l'appellation suivante: Clin d'œil à l'histoire de l'Aubrac, l'aligot - pomme de terre Institut de Beauvais (photo 4). Ainsi, dans un article intitulé "Tout l'Aubrac dans l'assiette», le journaliste J.-L. Galesne décrit ainsi 
l'attractivité du restaurant du Puech de Suquet : «On vient du monde entier rencontrer cet homme de foi qui a mis sa cuisine au service de la nature, et recompose dans l'assiette avec exactitude son image nourricière à partir d'ingrédients les plus divers, légumes (plus d'une centaine à l'année), herbes, fleurs, etc., extraits de la terre avec le plus grand soin » (Les Échos week-end, 6-7 septembre 2002).

Dans un second temps, Michel Bras va rendre les paysages de l'Aubrac admirables, désirables et « visibles » à l'échelle nationale et internationale. Il va communiquer sur leur singularité d'une part et sur leur irrésistible beauté d'autre part. Pour ce faire, il aime citer ce texte du poète local Henri Pourrat : «L'Aubrac, on y est dans l'air. Jamais, je n'ai eu ailleurs un tel sentiment d'être au milieu de l'air. Je ne sais pas, c'est le site, sans doute, ces longs pacages nus, et pas d'arbre, à peine de loin en loin de curieux hérissons de basalte; des bandes de montagnes et des bandes de vaches qui vaquent sans chien entre d'interminables cordons de pierres grises. C'est cela, et puis surtout, c'est ce cristal, ce goût cru du vent, d'herbes amères, d'eau de neige et ces rivières pavées où l'eau glacée de truites court sur les hexagones de basalte.» En tant que cuisinier, Michel Bras va alors mettre en exergue l'originalité et l'esthétique sans égale de l'animal emblématique qui est partie intégrante du paysage du plateau : le bovin de race Aubrac. Selon une documentation interne de l'Union Aubrac, «les animaux ont une robe unicolore variant du fauve au gris froment, plus foncée sur l'encolure pour les mâles. Les muqueuses, la pointe des cornes et le bout de la queue sont noirs. Le tour du mufle et de l'œil sont blancs soulignés de noir ${ }^{12}$ ». Pour le chef, les bovins de race Aubrac qui occupent la montagne ${ }^{13}$ éponyme ${ }^{14}$ produisent une viande "sincère » : " À un moment où la viande de bœuf est en perte d'identité, en Aubrac, les pâturages nourrissent des bêtes aux yeux ourlés de noir qui offrent une alternative rassurante quant à l'avenir de la filière viande de goût. Certifié par un Label Rouge, le bœuf fermier Aubrac offre une viande mûre qui renoue avec notre mémoire. Elle est belle à regarder, présentant une pigmentation rouge rubis, elle sent bon, offrant cette odeur franche pleine de promesses. Elle est goûteuse, proposant une texture, une mâche qui peut parfois déranger mais qui est ô combien rassurante sur son origine» (Bras, 2002). Créant ainsi un rapport organoleptique intérieur à la viande de bœuf, Michel Bras propose des plats emblématiques tels que : Condiments de fleurs, de légumes et d'anchois pour : le filet de boeuf - pure race Aubrac - rôti à la braise; tomates \& huile d'olive, des haricots Saint-Fiacre ou De pure race Aubrac, la pièce de bœuf rôtie: un jus de panais à la muscade \& touche de grillons; haricots mange-tout de Saint-Fiacre. Afin de valoriser ce rapport, le cuisinier propose aux hébergés de découvrir et de se forger leur propre vision de la beauté des paysages en les invitant à se promener avant le dîner: "Alors cette attention aux choses de la nature, ces découvertes, cette exaltation, ces escapades que nous aimons tant, nous avons envie de vous les faire partager. C'est pourquoi nous avons réuni autour de quelques thèmes, des rencontres qui vous permettront de vous imprégner à votre tour de l'Aubrac ${ }^{15}$. » Dans le même esprit, il a délibérément choisi d'orienter les tables du restaurant vers les larges baies vitrées qui permettent aux mangeurs de déguster les produits agricoles cuisinés et servis dans leur assiette tout en communiant directement avec les paysages qui les ont vu naître et en communiquant indirectement avec les producteurs qui les ont façonnés (Pitte, 1993). 
Photo 4 : Le service de l'aligot au restaurant Bras (cliché de l'auteur pris le 10 septembre 2008)/ Service of aligot (creamed potato with cheese) at the restaurant Bras (picture taken by the author on 10 September 2009)

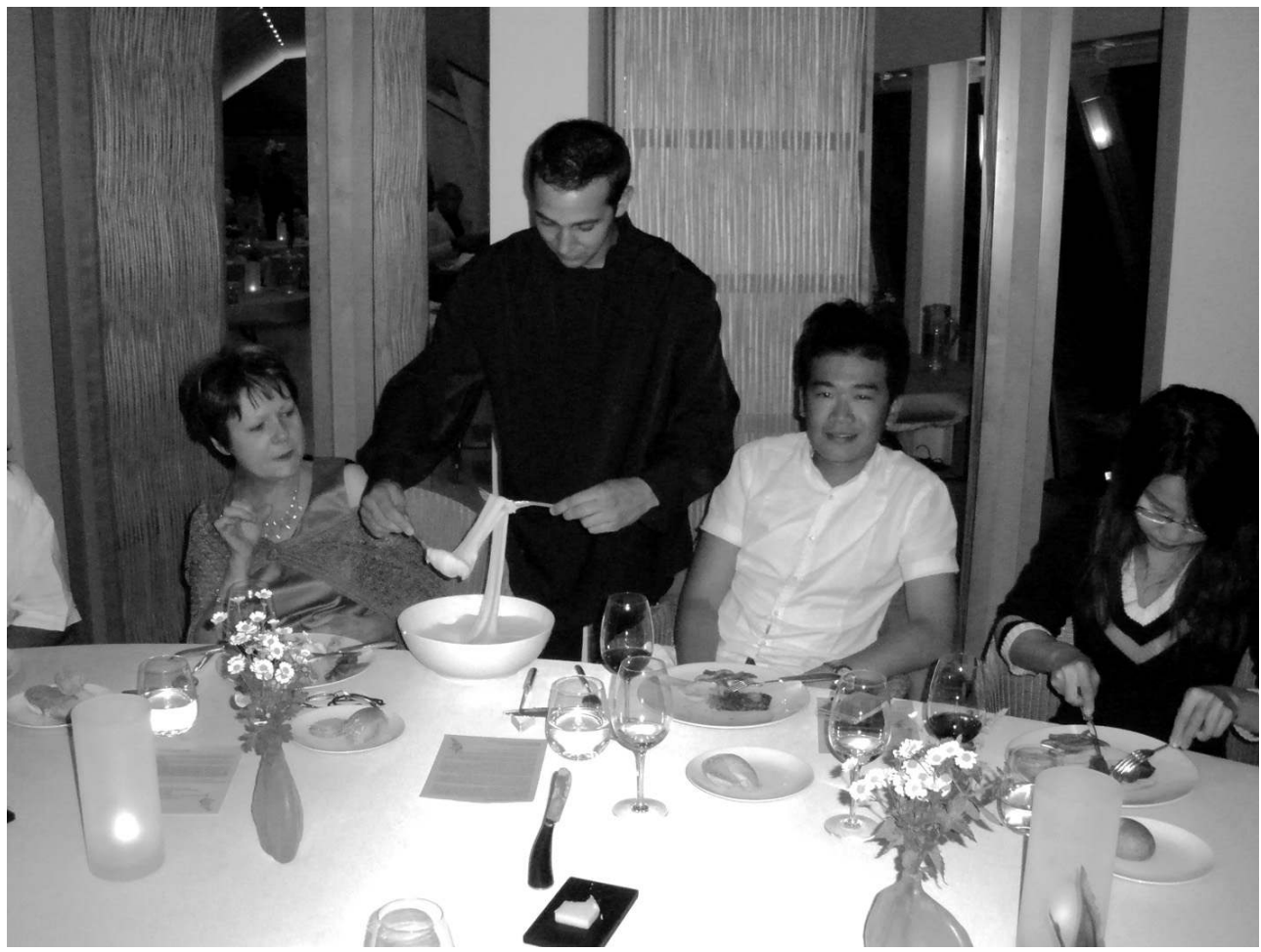

« Tourner » l'aligot demande une grande dextérité. En effet, une fois le fromage incorporé, l'aligot doit être battu suffisamment longtemps pour qu'il « file » en formant de longs rubans homogènes et souples. Si la préparation est trop battue, elle «casse ». Si elle est trop chauffée, elle granule et ne « file » plus. Plus l'aligot « file», plus il est réussi. C'est un plat de subsistance qui tient au ventre et « en sympathie » : il est souvent qualifié de « ruban de l'amitié ».

"Turning" aligot requires a great dexterity. Indeed, once cheese is blended, aligot needs to be beaten for long enough so that it goes stringy with long homogeneous ribbons. If preparation is too much beaten, ribbons break. If it is too much heated, it becomes granular and it does not go stringy anymore. The stringier aligot goes, the better it comes out. It is a nourishing convivial subsistence dish, often described as "friendship ribbon".

\section{Initier et encourager de nouvelles pratiques socio-économiques locales}

18 L'audacieuse stratégie de Michel Bras va constituer une initiation et un encouragement à la transformation qualitative et créative des pratiques des acteurs socio-économiques locaux.

\section{La sphère travail-emploi : miser sur la haute qualification et favoriser sa diffusion géographique à plusieurs niveaux scalaires}

19 L'existence du restaurant Bras a des retombées économiques qui se traduisent en termes de travail, d'emplois et de revenus principaux ou secondaires. En effet, la présence du restaurant implique des besoins théoriques et réels de main-d'œuvre, c'est 
la population techniquement nécessaire (Mathieu, 1995). Le projet de Michel Bras a ainsi induit la création :

- d'emplois directs : environ 65 emplois temporaires (durant les 7 mois d'ouverture annuelle) et 8 emplois permanents;

- d'emplois induits par le fonctionnement de l'entreprise au travers de son activité propre (approvisionnement, entretien, maintenance des équipements...) ;

- d'emplois indirects induits par la fréquentation de l'entreprise y compris auprès des autres hôteliers-restaurateurs $\mathrm{du}$ plateau de l'Aubrac (hébergement, restauration, accompagnement des visiteurs). agricole. En effet, il suscite des productions agricoles de qualité encouragées et guidées par le renouvellement gustatif et le foisonnement créatif cuisinier. L'effet d'entraînement vers une agriculture à objectif qualitatif est direct lorsque Michel Bras est personnellement client de l'agriculteur ou de l'éleveur et indirect lorsque ses exigences et ses préférences se diffusent auprès des producteurs dont il n'est pas le client. Constamment à l'affût, le cuisinier est à la recherche de produits agricoles de très grande qualité et "hauts" en goût. Le restaurant Bras joue alors un rôle polarisateur sur son environnement agricole pour l'approvisionnement en produits frais, divers (près de 250 variétés de légumes, herbes condimentaires et fleurs comestibles utilisées annuellement) et savoureux. Le cuisinier s'approvisionne en bœuf fermier Aubrac pure race élevé sur le plateau de l'Aubrac (à la boucherie Conquet de Laguiole), en fruits frais dans les vergers du Rougié (Marcillac, Pruines) et du Naucellois (Naucelle), en légumes frais dans la vallée du Lot (GAEC de Port d'Agrès), en fromages AOC sur la commune de Laguiole (Coopérative Jeune Montagne et GAEC de l'Ecir) et propose à sa carte le veau fermier label rouge d'Aveyron et du Ségala. 

motivée qu'il s'agisse du gibier: la biche de la forêt des Truques (point culminant de l'Aubrac aveyronnais avec ses $1469 \mathrm{~m}$ d'altitude) avec La pièce de biche de la forêt des Truques, poire William pochée au beurre et courge butternut, une touche de chutney à la rhubarbe et aux raisins, le jus; ou, bien sûr, les fleurs et les herbes odoriférantes du plateau avec le Méli-mélo de printemps: d'herbes, de légumes, de tourteau et de poisson, vinaigrette douce aux raisins (carte du restaurant). Son engagement pour la qualité des matières premières passe également par la réhabilitation de produits alimentaires prosaïques oubliés : les racines telles que le panais (Les noix de Saint-Jacques poêlées sur une purée de panais, le bouillon moussé au beurre, touche de maceron), les courges telles le potimarron (La mousse de potimarron relevée de café, de grué et de noix et Le biscuit tiède de potimarron coulant, an 97, crème glacée au café, potimarron confit et touche de café), la courge Jack Be Little (Dans une courge Jack Be Little, des coquillages et leur jus, "olives fortes", basilics parfumés et pain frit) ou la courge Sweet Dumpling (Sous un voile de noisettes, la crème de courge Sweet Dumpling, huile de noisettes et le laguiole jeune en crème), la châtaigne (La soupe de châtaignes paquette aux truffes de pays, feuilles d'épinard et huile d'amandes de pruneaux); et la promotion de produits locaux susceptibles d'obtenir une réputation gastronomique : les vins VDQS d'Entraygues et AOC Marcillac avec le Foie gras de canard poêlé, lie de Marcillac parfumée et touche de rau-ram, du coing, des raisins muscat et un jus de pomme akane (carte du restaurant).

Bien que rares et peu structurés, les circuits d'approvisionnement courts sont valorisés par Michel Bras qui permet ainsi le maintien une agriculture vivante pour laquelle on ne raisonne plus en termes de diminution du nombre d'emplois mais en termes d'innovation et d'initiative, de statut des membres de l'exploitation, de pluriactivité et de diversification agricole. Tout en privilégiant un approvisionnement local, c'est-àdire à l'échelle du plateau de l'Aubrac et, pour les légumes notamment, des vallées du Lot et de la Truyère, Michel Bras tisse un écheveau de relations avec les acteurs agricoles des terroirs aveyronnais et, plus largement du Sud-Ouest de la France (jusqu'à Saint Jean-de-Luz, pour certains poissons). Pour quelques produits spécialisés (épices, chocolats), il élargit encore le rayon géographique de ses filières d'approvisionnement à l'échelle mondiale (Brésil, Inde, Chine, etc.). Le développement agricole à objectif qualitatif s'adresse à un petit nombre d'éleveurs (alors que le modèle agricole dominant est l'élevage fondé sur de grosses exploitations familiales) et aux maraîchers. Au-delà des retombées économiques importantes, l'engouement des producteurs locaux pour les débouchés qu'offre ce marché est sous-tendu par la construction socioculturelle d'une renommée nationale voire internationale basée sur la connaissance et la promotion de produits typés et originaux. En effet, c'est une valorisation personnelle qu'ils réalisent en écoulant leurs produits qui fondent une identité qualitative et créative.

\section{Identifiant, identification et identités}

L'analyse des retombées de l'installation du restaurant Bras sur le développement local doit prendre en compte également les hésitations et les craintes qu'elle implique et qu'elle a entraînée. L'identification locale au restaurant Bras fut progressive. La mise en place des conditions nécessaires au développement local fut activée par la 
reconnaissance extérieure et passa par la construction progressive d'une identité productive et d'un contrôle socioculturel du territoire à caractère qualitatif et créatif.

\section{Des hésitation et craintes initiales...}

26 Le restaurant Bras ne constitua un identifiant pour les locaux qu'après la dissipation de quelques hésitations et craintes initiales. D'un côté, les acteurs politiques se montrèrent frileux devant l'ampleur et le caractère très innovant du projet. Cette hésitation politique entre facilité du conservatisme et inquiétude de la nouveauté fut lisible dans l'affaire de la délivrance du permis de construire qui suscita des polémiques. D'un autre côté, les acteurs économiques, les hôteliers-restaurateurs au premier chef, animés par des sentiments de jalousie et de frustration, se sentirent concurrencés et montrèrent quelques craintes. Elles sont révélatrices de la difficile inscription du restaurant à la tête d'un réseau professionnel et à son positionnement au sommet de la hiérarchie des hôtels-restaurants du plateau. Le restaurant Bras possède à la fois un rôle de moteur et de promoteur local mais, dans le même temps, il déploie aussi inévitablement son ombre portée. Cependant, l'identification collective pris consistance après l'obtention de la troisième étoile au guide Michelin en 1999 qui constitua un détonateur.

\section{...À la constitution d'une identité productive locale...}

Les réactions mettant fin aux hésitations et aux craintes initiales sont liées à la prise de conscience collective de l'avantageuse récupération des images et des discours construits par Michel Bras dans la succession, depuis les années 1960, des initiatives et des actions locales.

Les plus précoces furent basées sur la différenciation qualitative du lait et de la viande de bœuf initiées par un plan de conservation de la race bovine Aubrac. La mise en place de filières laitières et bouchères en relation avec la revalorisation de la race Aubrac débute en 1960 par la création à La Terrisse de la coopérative Jeune Montagne à l'initiative d'André Valadier, éleveur charismatique accompagné de 54 jeunes agriculteurs. Le fromage de Laguiole ${ }^{16}$ obtient l'appellation d'origine contrôlée (AOC) en 1961 (abrogation par décret le 29 décembre 1986). Dans le même esprit, l'Union Aubrac est créée en 1979. L'unité de sélection et de promotion de la race locale lance, en 1991, la marque commerciale «Fleur d'Aubrac " s'appliquant à des génisses d'embouche issues du croisement des races Aubrac et Charolaise. Cette dernière a obtenu une certification de conformité produit (CCP) et est engagé sur la voie de l'indication géographique protégée (IGP) «viande bovine du pays de l'Aubrac ». Michel Bras sera à la fois un ardent défenseur, un acteur à part entière, un héraut et un promoteur de ces deux filières agroalimentaires.

Plus récemment, et après une longue période durant laquelle les acteurs économiques et politiques locaux se sont évertués à doper la vente de couteaux de Laguiole à la provenance et à la qualité souvent douteuses, dans la même lignée, les acteurs de la filière du couteau de Laguiole, notamment La Forge de Laguiole (dirigée par André Bras, le frère de Michel) ont décidé de réagir en engageant une "relocalisation» de la production d'une part et une différenciation créative d'autre part en nouant des relations techniques avec des designers. En effet, la Forge de Laguiole, manufacture de 
couteaux, nait en 1987: "C'est l'aboutissement d'un projet de passionnés qui va relancer, à Laguiole, la fabrication du couteau de Laguiole. Le design de cette manufacture est confié à Philippe Starck et traduit parfaitement la philosophie de l'entreprise : allier tradition et création. L'architecture de verre et d'aluminium, d'où s'élance une lame de 18 mètres de haut, symbolise l'activité et le dynamisme de l'entreprise. Dès lors, toutes les étapes de la fabrication du couteau sont à nouveau réalisées sur son lieu d'origine. C'est la renaissance du couteau : le retour du laguiole à Laguiole. À Laguiole, la grande lame de Philippe Starck, sortie du toit de la manufacture Forge de Laguiole, semble découper les nuages et dessiner dans le ciel aveyronnais le "L" capital et majuscule qui signe tous les couteaux que l'on fait ici. Depuis, la Forge de Laguiole n'a de cesse de faire évoluer sa fabrication tout en respectant l'héritage du savoir-faire d'antan. Du petit couteau d'usage local, il est aujourd'hui devenu un objet culte, référence du design et synonyme d'art de vivre ${ }^{17}$. " Sur chacune des tables dressées, à la pointe du couteau de Laguiole, Michel Bras pose alors un petit carré de papier glacé et plié dans lequel il explique son attachement affectif à l'objet et l'importance symbolique de conserver le même couteau jusqu'à la fin du repas (à la différence des autres couverts qui eux sont changés à chaque assiette) : «Chez nous, l'ordonnancement de la table reposait sur un objet familier, qui se voulait pratique : le Laguiole. Ce couteau fermant a su acquérir ses lettres de noblesse. Offert à l'adolescent, il lui permettait d'être admis dans le monde des adultes. Depuis peu, il a rejoint notre mise de table dans un esprit résolument d'aujourd'hui. Christian Poumeyrol a su mettre en forme cet objet enraciné dans notre terroir que, enfant, je sculptais dans des branches de noisetier. Ce compagnon fidèle, ordinairement blotti au fond de la poche, accompagne nos hôtes durant le temps du repas pour partager un moment d'Aubrac. »

\section{...Au nécéssaire contrôle socioculturel du territoire}

Cette construction d'une identité productive qualitative et créative a récemment conduit les acteurs du plateau à contrôler et protéger les marques de territoire «Laguiole» et "Aubrac»: «Les noms de "laguiole" et "Aubrac" jouissent d'une incontestable notoriété, consacrée de très longue date et bien au-delà du terroir local. Cette renommée s'est forgée à travers entre autres un célèbre couteau, un fromage (le Laguiole AOC) une race bovine (la race Aubrac), ainsi qu'un plat traditionnel (l'Aligot de l'Aubrac), pour ne citer que ces produits de grande réputation [...] Comme toute dénomination dotée de notoriété et donc de valeur marchande, les noms "Laguiole" et "Aubrac" font ainsi l'objet de convoitises et par conséquent de multiples usurpations [...] Protéger les dénominations "Laguiole" et "Aubrac", étroitement liées de par la position centrale de la commune de Laguiole au sein de l'entité géographique Aubrac, s'avère être une nécessité pour la survie des produits de terroir. C'est pourquoi, il y a bientôt 3 ans, quelques couteliers ont décidé d'organiser une concertation autour d'un projet d'association à caractère interprofessionnel avec un seul mot d'ordre : défendre les dénominations de "Laguiole" et d'“Aubrac". Ainsi, depuis plus d'une année, avec l'appui d'Aveyron Expansion, les couteliers et la mairie de Laguiole étudient les moyens de préserver les intérêts de la coutellerie de Laguiole et plus largement d'autres secteurs économiques également concernés par la défense de ces dénominations. [...] Elle aura vocation à faire connaître et reconnaître les spécialités locales et défendre au besoin, par tous les moyens appropriés, y compris judiciaires, les dénominations "Laguiole" et "Aubrac" sur le territoire AOC de référence. Le 28 juin dernier à Laguiole, 
un certain nombre d'entreprises et de collectivités (fromage AOC, restaurant Michel Bras...) se sont donc retrouvées en présence de Jean Puech, Président du Conseil Général de l'Aveyron, en assemblée générale constitutive afin de sceller leur "union" contre toute utilisation abusive et usurpation des dénominations "Laguiole" et "Aubrac" [...]» (Conseil Général de l’Aveyron, 2007). Michel Bras est le vice-président de ce dispositif.

31 L'ouverture vers l'extérieur et la valorisation de l'échelle internationale conduisent à la production de copies et de contre façons, y compris dans le domaine culinaire. Ainsi, le biscuit de chocolat coulant ${ }^{18}$, dessert créé en 1982 et devenu emblématique, a connu d'infinies variations à travers le monde entier, telles que le fondant au chocolat, le gâteau mi-cuit et le moelleux au chocolat. A tel point que le cuisinier décida, en septembre 1999, de déposer le nom à l'Institut national de la propriété industrielle à Toulouse. C'est la raison pour laquelle Michel Bras a tenu à participer à cette initiative collective de contrôle « associatif » dont les limites territoriales sont celles du plateau. Dans ce cas, le développement local passe par la création d'une « marque » identitaire à effet local dont les retombées sont aujourd'hui impossibles à évaluer, faute d'une part d'une réelle stratégie collective d'application et d'autre part de son caractère imminent.

Dans le même esprit, vraisemblablement en relation avec l'esthétisation des paysages du plateau et de leur attractivité « gustative » réalisée par Michel Bras, la démarche de qualification territoriale Parc Naturel Régional Aubrac constitue, elle aussi, une forme d'expression de cette volonté collective de contrôle socioculturel du territoire.

\section{De l'identité productive à l'identité gustative locale}

Parallèlement, le dispositif gustatif de Michel Bras va conduire d'une part à un enrichissement des savoirs et des apprentissages gustatifs grâce à la créativité et aux " métissages » culinaires proposés et d'autre part au saisissement d'une singularité liée au goût enracinée : l'appétence pour le rance. Il s'agit d'un travail sur la mémoire collective gustative qui injecte de l'historicité et de l'identité dans la dynamique du développement local : la qualification d'un goût de " pays " (Etcheverria et Bras, 2004). Ainsi, la préférence et l'identification collective locales au rance est mise en exergue par Michel Bras: «Du nord-Aveyron: dans une croûte aux noix, les petits gris au beurre travaillé, oignon dit de florence poêlé, une huile rance comme une crème ou Toute la puissance de la mer : sur du chou-fleur râpé, ormeaux juste raidis, consommé de coquillages \& parfum de rance ou encore juste poêlé au jambon cru et au beurre : le homard émoustillé de paillettes/carcasse et de zestes, jeunes poireaux \& un bouillon rance, mandarine calamenthis » (carte du restaurant).

De même, le traitement culinaire du fromage de Laguiole est révélateur à la fois de l'appropriation collective du rance (valorisation du vieux Laguiole affiné) et de la créativité culinaire du cuisinier qui n'hésite pas à le valoriser dans la composition d'un dessert étonnant: le Millefeuille à la nougatine au beurre, crème fromagère au Laguiole et coulis au beurre noisette, qui rehausse les saveurs de fleurs de printemps et de noisette du fromage.

Cet enrichissement et ce saisissement sont amplifiés et confirmés par le classement des «burons de l'Aubrac» comme Site Remarquable du Goût (pour la dynamique agro- 
alimentaire, gastronomique et touristique du plateau) et de la commune de Laguiole comme Ville Gourmande en 2001.

\section{Conclusion}

La singulière trajectoire du plateau de l'Aubrac offre l'exemple d'un développement local qualifié par l'amour du lieu et de ceux qui l'habitent. Le développement local est animé, construit idéologiquement au travers de la constitution d'une image et d'un discours attrayants, rendus visibles à l'échelle internationale par un acteur « central ", le cuisinier Michel Bras, qui va ainsi induire un repositionnement géographique relatif de «son plateau " et assurer son rayonnement extérieur. Il montre en effet une remarquable capacité à jouer avec les niveaux scalaires et à envisager les emboitements d'échelles géographiques. Le développement local proposé est donc spécialisé, à objectif qualitatif et créatif. Il est fondé sur la diversité et la diversification des activités socioéconomiques autour de l'alimentaire et du goût. Il s'exprime par un ensilage d'initiatives individuelles plus que par une réelle collaboration collective. La dimension communicative est éminemment importante. Depuis l'obtention de la troisième étoile au guide Michelin, il passe par l'esquisse, notamment à travers les marques identitaires " associatives ", d'un réseau au sens développé par C. Neuschwander : «Un assemblage variable d'acteurs libres qui entretiennent entre eux une communication forte. Le réseau est d'abord communication » (Neuschwander, 1991).

Le restaurant Bras constitue l'un des puissants ressorts de cette vitalité. Il a rompu le cercle vicieux de l'isolement et de la fragilisation et a participé à la mise en place des conditions nécessaires au développement local. Il montre ainsi des effets géographiques, socio-économiques et culturels intéressants pour cet espace rural non polarisé : il change les qualités et valorise le lieu, active une image de marque et diffuse un discours positifs et rayonnants. Il conduit à la recomposition d'une identité productive et gustative qui autorise l'insertion du cuisinier dans le système local d'acteurs et l'articulation de sa stratégie individuelle aux dynamiques socioéconomiques à l'œuvre.

L'analyse des effets du restaurant Bras sur la mise en place des conditions nécessaires au développement local active la question de la durabilité d'une dynamique reposant sur la "centralité » et le charisme si original d'une seule figure, certes devenue emblématique du plateau: Michel Bras. Quelle est alors la pérennité de cette dynamique au moment où Michel Bras se prépare à se retirer des fourneaux? Quelle place peut prendre son fils Sébastien qui a rejoint son père en cuisine depuis six ans? Car si Michel Bras fut le fondateur du restaurant, il en est aujourd'hui le passeur. Comment cette transmission de patrimoine (au sens strict), de savoir-faire, de valeurs, d'images et de discours fortement ancrés et éminemment personnels peuvent-ils s'inscrire dans les caractéristiques d'un développement local durable? 


\section{BIBLIOGRAPHIE}

BARBEy A., 1991. Midi-Pyrénées, Guide Bleus, Paris, Hachette, 1989, p. 187.

BRAS M., 1999. Le souffle de l'Aubrac, in L'Aubrac, guide randonnée, Clermont-Ferrand, Chamina.

BRAS M., 2002. Bras, Laguiole, Aubrac, France, Rodez, Éditions du Rouergue, 239 p.

BESSIÈRE J., 2001. Valorisation du patrimoine gastronomique et dynamiques de développement territorial.

Le haut plateau de l'Aubrac, le pays de Roquefort et le Périgord noir. Paris, L'Harmattan, 365 p.

CNAC, 2000. Inventaire du patrimoine culinaire de Midi-Pyrénées, Paris, Belin, 328 p.

COLLECTIF, 1970. L'Aubrac. Etude ethnologique, linguistique, agronomique et économique d'un établissement humain, Paris, Editions du CNRS, 7 tomes.

CONSEIL GÉNÉRAL DE L'AVEYRON, 2007. Laguiole et Aubrac défendent leurs dénominations, Lettre de l'économie en Aveyron, newsletter Aveyron Expansion, n² 20, Conseil Général de l'Aveyron, p. 1.

GALESNE J.-L., 2002. Tout l'Aubrac dans l'assiette, Les Echos week-end, vendredi 6 et samedi 7 février.

ETCHEVERRIA O., BRAS M. 2005. Existe-t-il un goût de l'Aubrac? in Géographie des saveurs, Revue Géographie et Cultures, L'Harmattan, n50, p. 63-76.

FUMEY G., ETCHEVERRIA O. 2004. L'Atlas mondial des cuisines et des gastronomies, Paris, Autrement, $79 \mathrm{p}$.

LAURENS L., 1999. Le long parcours de l'Aubrac... Productions de qualité, développement local et affirmation d'une identité micro-régionale. Sud-Ouest européen, Toulouse, Presses Universitaires du Mirail, $n^{\circ}$ 6, p. 51-60.

MATHIEU N. 1995. L'emploi rural. Une vitalité cachée, Paris, L'Harmattan, coll. « Alternatives rurales $", 183 \mathrm{p}$.

Mialon P., 1999. Désir d'Aubrac ou le désarroi des arpenteurs, Rodez, Editions Le Temps qu'il fait, $80 \mathrm{p}$.

NeUSCHWANDER C., 1991. Les réseaux et les acteurs : réflexion sur l'administration et le changement, in Le clair obscur des réseaux, Paris, L'Harmattan, coll. « Pour ».

PITTE J.-R., 1993. Des productions de qualité dans un paysage de qualité : un défi pour le monde rural français, L'aménagement foncier agricole et rural, 79, p. 19-22

SUBERVIE G., 1953. Chronique gourmande, Revue du Rouergue, Rodez, Imprimerie Carrère.

\section{NOTES}

1. Sur la définition du «grand» restaurant, se référer à l'introduction du dossier thématique.

2. Lou Mazuc est le nom aveyronnais du buron, cabane située dans les pacages (les estives) où les buronniers fabriquent le fromage de Laguiole.

3. Extrait du texte introduisant le menu du dîner du 10 septembre 2009, à l'occasion du voyage d'étude des étudiants du MASTER 1 Gastronomie et développement local de 
l'université d'Angers (UFR Ingénierie du Tourisme, du Bâtiment et des Service ESTHUA).

4. L'écir est le nom du vent local.

5. Site internet du restaurant Bras.

6. Document de présentation de l'hôtel-restaurant proposé aux clients et déposé sur les lits.

7. Plat aveyronnais ancien, le gargouillou est un ragoût de pommes de terre mouillé d'eau et agrémenté d'une tranche de jambon cru de montagne. Michel Bras l'a transformé en un ragoût de jeunes légumes relevé d'herbes et de fleurs sauvages et de graines germées. Il est devenu un plat fétiche.

8. Le haut plateau de l'Aubrac est exceptionnellement riche d'une flore comptant entre 1800 et 2000 plantes qui composent les herbages.

9. Il s'agit d'une Recherche Coopérative sur Programme (RCP) réunissant une équipe de chercheurs de diverses disciplines (ethnologues, sociologues, agronomes géographes, économistes) et visant à réaliser «une étude pluridisciplinaire d'un établissement humain ».

10. Il s'agit du caillé pressé, non salé et légèrement fermenté.

11. L'aligot était servi par les religieux du plateau aux pèlerins en route vers SaintJacques-de-Compostelle qui demandaient le couvert à la dômerie d'Aubrac. L'ancienne voie romaine, le via agrippa, qui leur servait de chemin passait par la vaste hêtraie habitée de loups et de sangliers qui recouvrait alors le plateau.

12. Site internet de l'UPRA Aubrac. Dernière consultation janvier 2011.

13. Précédé de l'article défini singulier, le mot montagne (et non plateau) désigne les monts d'Aubrac et, plus précisément, les pâtures situées à une altitude supérieure à 1 000 mètres.

14. Selon une " particularité " soulignée dans l'Inventaire du patrimoine gastronomique de la région Midi-Pyrénées, il s'agit d'une race "rustique qui vit en symbiose avec le milieu naturel de l'Aubrac qui lui a donné son nom. Elle est bien adaptée et supporte les fortes amplitudes climatiques entre été et hiver " (CNAC, 2000). La viande de bœuf d'Aubrac est réputée depuis le $\mathrm{XVIII}{ }^{\mathrm{e}}$ siècle. Il s'agissait initialement d'un bœuf engraissé durant l'hiver et le printemps pour être abattu à Pâques.

15. Document de présentation de l'hôtel-restaurant proposé aux hébergés et déposé sur les lits.

16. Selon l'article 2 du décret, il s'agit d'un "fromage à croûte séchée, fabriqué exclusivement avec du lait de vache cru et entier, emprésuré, à pâte ferme, non cuite, deux fois pressée avec broyage du caillé entre les deux pressages, salée et fermentée contenant au minimum 45 grammes de matière grasse pour 100 grammes de fromage. La durée de l'affinage est de quatre mois au minimum à compter de la date de fabrication. L'affinage et la conservation des fromages sont effectués à une température n'excédant pas $14^{\circ} \mathrm{C} »(1961)$. Son goût unique et inimitable légèrement anisé serait dû à la consommation de cistre par le cheptel bovin laitier.

17. Site internet de la Forge de Laguiole. Dernière consultation janvier 2011.

18. C'est en 1982 que Michel Bras met au point, après deux années de tâtonnement, son désormais célèbre biscuit de chocolat coulant. C'est un gâteau chaud et rond au cacao enserrant un noyau central de ganache chocolatée qui coule dans l'assiette dès le 
premier coup de cuillère. La technicité du cuisinier consista à définir l'épaisseur la plus appropriée du biscuit et le diamètre idéal du noyau. Imaginé en hiver, alors que le plateau de l'Aubrac est tapissé de neige et battu par l'écir, ce dessert se veut réconfortant à la manière du chocolat chaud préparée par la maman de Michel au retour du ski.

\section{RÉSUMÉS}

La singulière trajectoire du plateau de l'Aubrac montre le cas d'un espace rural, hâtivement qualifié de «marge» (CNRS, 1970), qui présente un développement socio-économique à contresens des évolutions prévues pour les zones de faible densité. En effet, il se caractérise par sa résistance à la désertification, par son usage social original et dynamique du territoire et par sa vitalité nonobstant son isolement géographique. Le (grand) restaurant est, semble-t-il, l'un des puissants ressorts de cette vitalité par l'ouverture vers l'extérieur qu'il assure et le rayonnement international qu'il permet. Ainsi, il a rompu le cercle vicieux de l'isolement et de la fragilisation et a participé à la mise en place des conditions nécessaires au développement local en se positionnant judicieusement dans le système local d'acteurs. L'exemple du restaurant Bras à Laguiole, triplement étoilé au guide Michelin, témoigne d'un développement local animé par la «centralité » d'un cuisinier, de la réussite de pratiques économiques et d'un contrôle socioculturel du territoire fondés sur la qualité et la créativité.

The peculiar path of the Aubrac plateau shows the case of a rural area, hastily described as "fringe" (CNRS, 1970), with a socioeconomic development opposite to the evolutions foreseen for low-density areas. Indeed, it is characterized by its resistance to depopulation, its original dynamic and social use of territory and its vitality notwithstanding its geographical isolation. Apparently, the (grand) restaurant is one of the impulses to this vitality. It has broken off the vicious circle of isolation and weakening, and it has contributed to the setting up of the necessary conditions for local development. The example of the Bras restaurant in Laguiole, awarded three stars by the Michelin, shows a local development led by the "centrality" of a chefrestaurateur, the success of economic practices and a social control of territory based on quality and creativity.

\section{INDEX}

Mots-clés : développement local, restauration, centralité, qualité

Keywords : local development, catering, centrality, quality 
AUTEUR

OLIVIER ETCHEVERRIA

UMR ESO (Espaces et SOciétés) 6590 CNRS ESO Angers (CARTA) (Université d'Angers), 5 bis Boulevard Lavoisier - 49045 Angers Cédex, France olivier.etcheverria@univ-angers.fr 